\title{
Kolorektales Karzinom: Gensignatur unterstützt Therapieentscheidung
}

\author{
Etwa jeder vierte Patient mit einem nodal-negativen, invasiven kolorektalen \\ Karzinom entwickelt nach der Exzision in kurativer Intention ein Rezidiv, \\ häufig mit letalem Ausgang. Erstmals wurde jetzt ein Prädiktionsmodell auf \\ der Basis von Gensignaturen vorgestellt, das Patienten mit potenzieller \\ Eignung für eine adjuvante Chemotherapie identifiziert.
}

eder vierte Patient mit einem invasi-
ven kolorektalen Karzinom (CRC) ohne diagnostizierte Lymphknotenbeteiligung entwickelt nach dem chirurgischen Eingriff ein Rezidiv und hat damit ein ähnlich hohes Mortalitätsrisiko wie CRC-Patienten in fortgeschritteneren, metastasierten Stadien. Klinisch relevante, molekulare prognostische Marker könnten die dem Tumor inhärente Aggressivität vorhersagen helfen. Die Autoren stützten sich auf die Version „Evolver", eine „Gen“-Software, zum Einsatz für eine Trainings/Test- und externe Validierungsphase. Ziel: Erstellung einer Gensignatur der Tumorzellen als klinisch nützliches Vorhersage-Modell (v. a. Rezidivrisiko) für Patienten mit einem nodal-negativen invasiven CRC der Stadien I und II. Als Proben dienten archivierte Formalin-fixierte, in Paraffin eingebettete primäre Karzinomgewebe von 74 Patienten mit CRC für das Trainings/ Test-Setting und insgesamt 264 Gewebeproben von Patienten aus 18 internationalen Zentren für die externe Validierung. Keiner der Patienten hatte eine neoadjuvante oder adjuvante Therapie bekommen. Zur Analyse fütterten die Autoren die Software mit den Expressionsprofilen von 225 vorher spezifizierten Tumor-Genen und leiteten daraus eine „36-Monats-Risikosignatur“ ab.

Die externe Validierung des Expressionsprofils von fünf tumorassoziierten Genen (OncoDefender-CRC) mit Bezug auf die Prognose ergab eine korrekte Klassifizierung für 62 von 92 RezidivPatienten und 87 von 172 Patienten ohne Rückfall. Hochrisiko-Patienten entwickelten mit höherer Wahrscheinlichkeit ein Rezidiv innerhalb von 36 Monaten als Patienten mit einem per Gensignatur ermittelten niedrigeren Risiko.

Fazit: Nach Kenntnis der Autoren liegt mit ihrem Prädiktionsmodell für das Rezidivrisiko von nodal-negativen CRCPatienten das erste validierte molekulare Prognose-Tool für CRC Stadium I und Kolonkarzinom Stadium II vor, das den standardisierten klinisch-pathologischen Prognosekriterien überlegen ist. Diese Gen-Signatur identifiziert Patienten mit einem erhöhten Rezidivrisiko innerhalb von drei Jahren nach der kurativen Chirurgie und eröffnet die Chance für eine individuell angepasste adjuvante Chemotherapie. Wolfgang Zimmermann

Lenehan PF et al. Generation and external validation of a tumor-derived 5-gene prognostic signature for recurrence of lymph node-negative, invasive colorectal carcinoma. Cancer. 2012;118(21):5234-44.

\section{CRC: Hyperinsulinämie und Tumorprogression}

\section{Die Ernährungsgewohnheiten von Darmkrebs-Patienten nach erfolgreicher Therapie beeinflussen offenbar das Rezidivrisiko und die Mortalität.}

\footnotetext{
räklinische und klinische Daten weisen auf einen positiven Zusammenhang zwischen Hyperinsulinämie und der Entwicklung eines kolorektalen Karzinoms hin. Viele der bekannten Risikofaktoren wie Übergewicht oder mangelnde sportliche Aktivität wirken sich direkt auf den systemischen Insulinspiegel aus. Ebenso führen Nahrungsmittel mit einem hohen glykämischen Index (GI) zu erhöhter Insulinausschüttung. Der GI bezeichnet ein Maß für die Höhe des Blutglukose-Spiegels nach Zufuhr von $50 \mathrm{~g}$ verwertbaren Kohlenhydraten mit einem Testlebensmittel (nach DGE, Ernährungs-Umschau. 2004;51(3):84). Die glykämische Last (GL), errechnet aus dem
}

Produkt des GI und der verwertbaren Kohlenhydratmenge (in Gramm) pro Lebensmittelportion dividiert durch 100, erlaubt eine qualitative und quantitative Beurteilung der glykämischen Antwort.

Jüngste Studien legen einen Zusammenhang zwischen Hyperinsulinämie und dem Rezidiv- und Mortalitätsrisiko von Darmkrebspatienten nach überstandener Therapie nahe. Die Autoren rekrutierten 1.011 Patienten mit einem Kolonkarzinom, Stadium III, aus einer Studie der Cancer and Leukemia Group B (CALGB 89803) für ihre prospektive Beobachtungsstudie. Sie werteten die Angaben der Patienten über ihre Ernährungsgewohnheiten während und sechs Monate nach Teilnahme an einer adjuvanten Chemotherapie aus und berechneten in einem statistischen Modell den Einfluss der GL, des GI sowie des Fructose- und Kohlenhydrat-Konsums auf die Rezidivrate und Mortalität.
In dieser Kohorte von Patienten mit einem Kolonkarzinom des Stadiums III, die postoperativ eine adjuvante Chemotherapie durchlaufen hatten, korrelierten eine erhöhte GL und Kohlenhydrataufnahme mit einem höheren Rezidivrisiko und niedrigerem Gesamtüberleben. Weiteres Ergebnis: Patienten mit einem erhöhten Body-Mass-Index (über $25 \mathrm{~kg}$ / $\mathrm{m}^{2}$ ) entwickelten signifikant früher ein Rezidiv als Normalgewichtige.

Fazit: Faktoren, welche die Energiebilanz beeinflussen, spielen offenbar eine wichtige Rolle bei der Tumorprogression von Patienten mit einem erfolgreich therapierten Kolonkarzinom im fortgeschrittenen Stadium. Diese Ergebnisse könnten dazu beitragen, durch Anpassung des Ernährungsverhaltens das Gesamtüberleben dieser Patienten zu verbessern.

Wolfgang Zimmermann

Meyerhardt JA et al. Dietary glycemic load and cancer recurrence and survival in patients with stage III colon cancer: findings from CALGB 89803. J Natl Cancer Inst. 2012;104(22):1702-11. 